\title{
Clinically suspected recurrence of gastric carcinoid proved to be hypocomplementaemic urticarial vasculitis syndrome with pulmonary involvement
}

Christoffer Sjöwall, Martin Hallbeck and Per Sandström

\author{
Linköping University Post Print
}

\section{Tweet}

N.B.: When citing this work, cite the original article.

Original Publication:

Christoffer Sjöwall, Martin Hallbeck and Per Sandström, Clinically suspected recurrence of gastric carcinoid proved to be hypocomplementaemic urticarial vasculitis syndrome with pulmonary involvement, 2015, Scandinavian Journal of Rheumatology, (44), 4, 337-339.

http://dx.doi.org/10.3109/03009742.2015.1020068

Copyright: Informa Healthcare http://informahealthcare.com/

Postprint available at: Linköping University Electronic Press http://urn.kb.se/resolve?urn=urn:nbn:se:liu:diva-121328 
Title: Clinically suspected recurrence of gastric carcinoid proved to be hypocomplementemic urticarial vasculitis syndrome with pulmonary involvement

Authors: Christopher Sjöwall ${ }^{1 *}$, Martin Hallbeck², Per Sandström³ ${ }^{3}$

1. Rheumatology/AIR, Department of Clinical and Experimental Medicine, Faculty of Health Sciences, Linköping University,

2. Pathology, Department of Clinical and Experimental Medicine, Faculty of Health Sciences, Linköping University,

3. Surgery, Department of Clinical and Experimental Medicine, Faculty of Health Sciences, Linköping University, Linköping, Sweden.

\section{* Corresponding author:}

Rheumatology Unit, University Hospital, SE-581 85 Linköping, Sweden

E-mail address: christopher.sjowall@liu.se

Telephone +46101032416

Fax: +46101031844

Running head: HUVS in a patient with previous carcinoid

Key words: Anti-C1q; Complement proteins; Neuroendocrine tumour; Urticaria; Vasculitis 


\section{LETTER/CASE REPORT}

Since first described in 1973, hypocomplementemic urticarial vasculitis syndrome (HUVS) has been recognized as a specific autoimmune disorder involving at least 6 months of urticaria with hypocomplementaemia in the presence of systemic manifestations e.g. arthritis/arthralgia, glomerulonephritis, uveitis and recurrent abdominal pain [1]. Skin biopsy is essential in the diagnosis of HUVS and the presence of anti-C1q antibodies has been judged "a rule without exception" [2], although the latter was recently questioned in a French study [3]. Herein we highlight a case with a history of gastric carcinoid and an elusive presentation of HUVS.

A Caucasian woman born in 1950 previously diagnosed with type-1 diabetes since 17 years of age (mild retinopathy and nephropathy), hypertension, hypothyroidism (high levels of anti-TPO antibodies), pernicious anaemia and former smoker sought acute care with abdominal pain, diarrhoea and fainting in July 2008. Infection was ruled out, and a computed tomography (CT) scan showed cholecystolithiasis only. Gastroscopy was performed; multiple broad-based polyps were found in the fundus and biopsies were taken. The biopsies showed chronic atrophic gastritis with carcinoid, positive for chromogranin A and synaptophysin, whereas glucagon, gastrin and serotonin staining were negative. Ki67 levels were below 1\% and the mitotic rate was 1 per 10 high-power field (Fig. 1, A-E). Blood analysis revealed elevated chromogranin A (33 nmol/L; reference $<4)$ and gastrin $(1510 \mathrm{pmol} / \mathrm{L}$; reference <60). Altogether, the carcinoids were defined as GEP-NET grade 1, type 1 according to the 2010 WHO/ENETS classification [4]. Whole-body CT scan found 2 liver lesions in the right liver, segment 5 and 7, but no signs of enlarged lymph nodes or visible gastric tumour. The liver tumours were 
characterised as one haemangioma and the second uncertain. As the stomach polyps were not possible to treat locally, the multidisciplinary conference recommended gastrectomy with lymph node dissection and perioperative liver ultrasonography. The operation was uncomplicated with gastrectomy, splenectomy and cholecystectomy, the liver lesions were judged as benign, and the postoperative course uneventful. The pathology report showed the same characteristics as the biopsies. No lymph node metastases were found. Blood chromogranin A and gastrin returned to normal levels postoperatively.

During the following 3 years the patient was affected by several allergic reactions with tachycardia and skin rash requiring oral betamethasone and intramuscular clemastine. Eventually, she developed pleuritis (visualised by X-ray), pericarditis (echocardiography), arthritis and persistent skin rash. Paraneoplastic syndrome or recurrence of gastric carcinoid was initially suspected, but judged unlikely due to a normal octreotide scan and low chromogranin A levels. Although the patient was ANA negative, she was referred to rheumatologist in November 2013 on the suspicion of systemic lupus erythematosus (SLE). At the same time, the dermatologist described the rash as urticaria-like on the trunk with palpable purpura on the lower extremities; and she had signs of systemic inflammation with elevation of CRP $95 \mathrm{mg} / \mathrm{L}$ and ESR 27 $\mathrm{mm} / \mathrm{h}$. Skin biopsy, unfortunately performed without immunofluorescence (IF) microscopy, from the left leg showed widespread purpura and leukocytoclasia, perivascular infiltrates composed of neutrophils without thrombi, necrosis or panniculitis (Fig. 1, F-G). The patient was prescribed topical betamethasone. 
In February 2014, the rheumatologist found family history of rheumatic diseases negative, and she had had one successful pregnancy and denied miscarriages. The arthralgia had disappeared with treatment of ibuprofen. However, a recent CT scan had confirmed $2.5 \mathrm{~cm}$ unilateral pleural effusion (left side) and at least $1 \mathrm{~cm}$ pericardial effusion. Blood analyses showed low haemoglobin $95 \mathrm{~g} / \mathrm{L}$ with no other cytopenias, CRP $<10 \mathrm{mg} / \mathrm{L}$, ESR $11 \mathrm{~mm} / \mathrm{h}$, urate $238 \mu \mathrm{mol} / \mathrm{L}$, creatinine $71 \mu \mathrm{mol} / \mathrm{L}$ and no $\alpha 1$-antitrypsin deficiency. Urinalysis indicated microhaematuria, but no cellular casts. ANA test by IF was negative, as were tests for ANCA and anti-phospholipid antibodies. However, complement proteins C3 (0.39 g/L; range 0.70-1.3) and C4 (0.12 g/L; range 0.13-0.32) and the classical complement function, assessed by a haemolytic assay (37\%; range 80120), were significantly reduced. Anti-C1q antibodies were clearly positive (73 units/L; range $<26$ ) by ELISA, and were further confirmed positive with Western blot. The patient was subsequently referred to pulmonologist who performed a diagnostic pleuracentesis and spirometry. Malignant cells were not found. The spirometry displayed reduced FEV-1 (56\% of reference), reduced VC (62\% of reference), TLC within normal range, and diffusion capacity reduced ( $48 \%$ of reference). The diagnosis of chronic obstructive pulmonary disease related to HUVS was set.

This case report illustrates a patient with a high autoimmune burden (type-1 diabetes, hypothyroidism, pernicious anaemia) who develops a rare malignancy with an annual incidence rate of 2.4 per 100.000 women [4], and eventually another uncommon rheumatic disease with involvement of skin, joints, lungs and serosa. The most important differential diagnosis to exclude was paraneoplastic syndrome [5]. Although HUVS has been described in association with malignancies [6,7], we consider the connection uncertain herein. Pulmonary involvement with is frequent in HUVS and 
constitutes a leading cause of morbidity and mortality [8]. Clinical manifestations and serologies in HUVS and SLE are mostly adjacent [2], but in this case the patient did not meet the recent and sensitive SLE classification criteria $[9,10]$. Intriguingly, the target epitope of anti-C1q found in HUVS is usually different from that of anti-C1q in SLE, and this can be used to differentiate the two conditions [11]. Anti-C1q primarily recognizes the conformationally changed collagenous part of C1q in SLE, and is detectable by ELISA only. In HUVS, however, anti-C1q typically targets native C1q and is found by both ELISA and Western blot.

\section{Acknowledgements}

Dr. Jonas Wetterö is acknowledged for comments on the manuscript. CS received grants for this work from the Swedish Society for Medical Research and the County Council of Östergötland. MH was financed by the Swedish Research Council (VR), grant No. 5232013-2735. 


\section{References}

1) McDuffie FC, Sams WM Jr, Maldonado JE, Andreini PH, Conn DL, Samayoa EA. Hypocomplementemia with cutaneous vasculitis and arthritis. Possible immune complex syndrome. Mayo Clin Proc 1973;48:340-48

2) Kallenberg CG. Anti-C1q antibodies. Autoimmunity Rev 2008, 8:612-15

3) Jachiet M, Flageul B, Deroux A, Le Quellec A, Maurier F, Cordoliani F, et al. The clinical spectrum and therapeutic management of hypocomplementemic urticarial vasculitis: data from a French nationwide study of 57 patients. Arthritis Rheumatol 2015:67:527-344

4) Niederle MB, Hackl M, Kaserer K, Niederle B. Gastroenteropancreatic neuroendocrine tumours: the current incidence and staging based on the WHO and European Neuroendocrine Tumour Society classification: an analysis based on prospectively collected parameters. Endocr Relat Cancer 2010;17:909-18

5) Manger B, Schett G. Paraneoplastic syndromes in rheumatology. Nat Rev Rheumatol 2014;10:662-70

6) Wilson D, McCluggage WG, Wright GD. Urticarial vasculitis: a paraneoplastic presentation of B-cell non-Hodgkin's lymphoma. Rheumatology (Oxford) 2002;41:476

7) Jamison SC, Brierre S, Sweet J, de Boisblanc B. A case of precocious emphysema and lung cancer in a woman with a history of hypocomplementemic urticarial vasculitis. Chest 2008;133:787-89

8) Schwartz HR, McDuffie FC, Black LF, Schroeter AL, Conn DL. Hypocomplementemic urticarial vasculitis: association with chronic obstructive pulmonary disease. Mayo Clin Proc 1982;57:231-8

9) Petri M, Orbai AM, Alarcón GS, Gordon C, Merrill JT, Fortin PR, et al. Derivation and validation of the Systemic Lupus International Collaborating Clinics classification criteria for systemic lupus erythematosus. Arthritis Rheum 2012;64:2677-86

10)Ighe A, Dahlström Ö, Skogh T, Sjöwall C. Application of the 2012 Systemic Lupus International Collaborating Clinics classification criteria in a regional Swedish systemic lupus erythematosus register. Arthritis Res Ther 2015;17(1):3

11)Mårtensson U, Sjöholm AG, Sturfelt G, Truedsson L, Laurell AB. Western blot analysis of human IgG reactive with the collagenous portion of C1q: evidence of distinct binding specificities. Scand J Immunol 1992;35:735-44 


\section{Figure legend}

Figure 1. Histology of the gastric (A-E) and skin (F-G) biopsies. A-B. Haematoxylin-Eosin staining showing typical morphology of a gastric GEP-NET grade 1. The diagnosis was confirmed by the expression of broad spectrum cytokeratine (CKAE1/3; C), synaptophysin (D) and chromogranin A (E). F-G. Haematoxylin-Eosin staining showing extensive purpura with leukocytoclastic vasculitis. Scale bar: A, F = $100 \mu \mathrm{m}, \mathrm{B}, \mathrm{G}=25$ $\mu \mathrm{m}, \mathrm{C}, \mathrm{D}, \mathrm{E}=37 \mu \mathrm{m}$. 


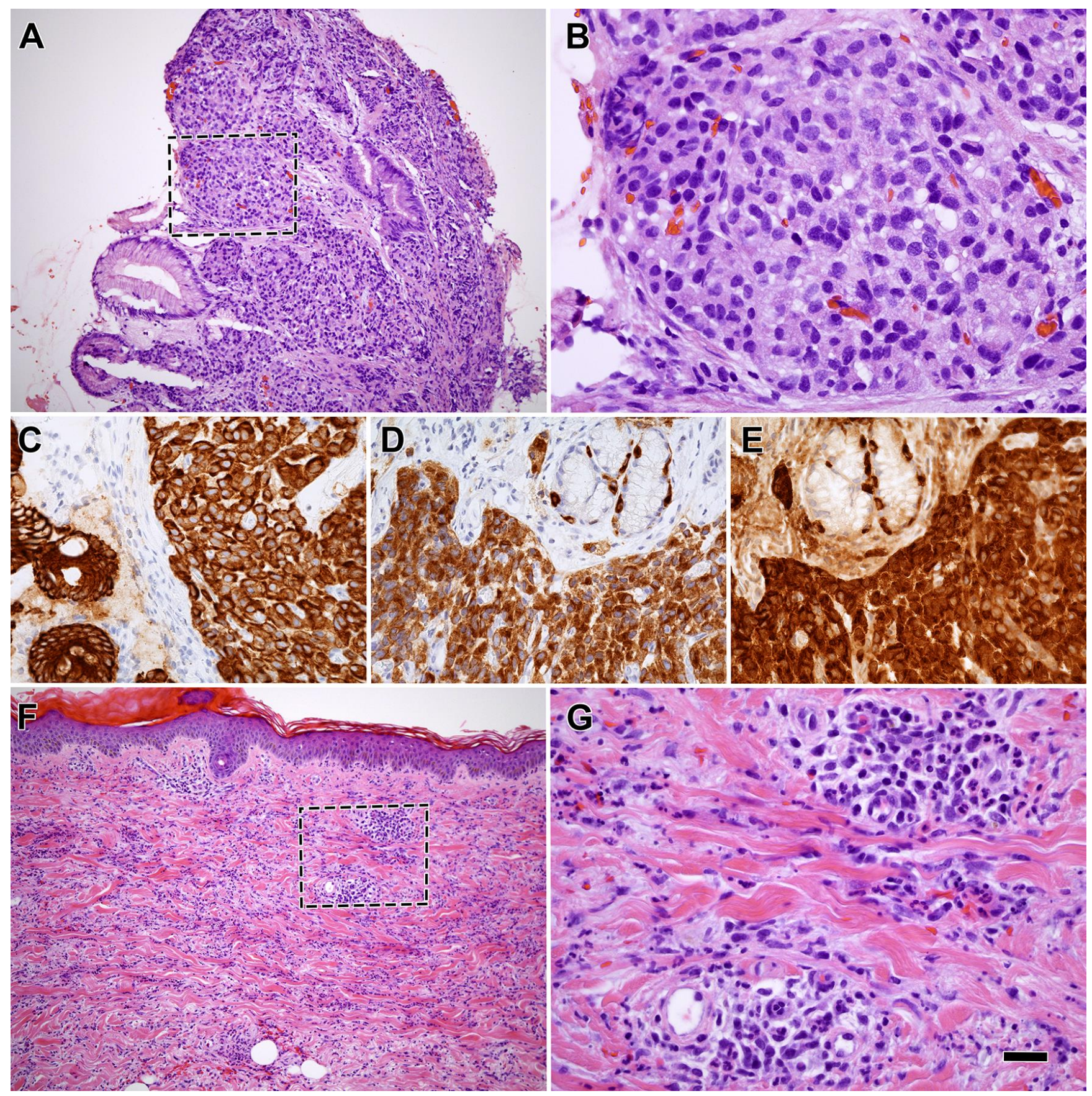

Figure 1 